Draft Version June 3, 2022

Preprint typeset using $\mathrm{L}^{\mathrm{A}} \mathrm{T} \mathrm{E}$ X style emulateapj v. 01/23/15

\title{
CARBON CHAINS AND METHANOL TOWARD EMBEDDED PROTOSTARS
}

\author{
Dawn M. Graninger \\ Harvard-Smithsonian Center for Astrophysics, Cambridge, MA, 02138 \\ Olivia H. Willins ${ }^{1}$ \\ Harvard-Smithsonian Center for Astrophysics, Cambridge, MA, 02138 \\ AND \\ KARIN I. ÖBERG \\ Harvard-Smithsonian Center for Astrophysics, Cambridge, MA, 02138 \\ Draft version June 3, 2022
}

\begin{abstract}
Large interstellar organic molecules are potential precursors of prebiotic molecules. Their formation pathways and chemical relationships with one another and simpler molecules are therefore of great interest. In this paper, we address the relationship between two classes of large organic molecules, carbon chains and saturated complex organic molecules (COMs), at the early stages of star formation through observations of $\mathrm{C}_{4} \mathrm{H}$ and $\mathrm{CH}_{3} \mathrm{OH}$. We surveyed these molecules with the IRAM $30 \mathrm{~m}$ telescope toward 16 deeply embedded low-mass protostars selected from the Spitzer $c 2 d$ ice survey. We find that $\mathrm{CH}_{3} \mathrm{OH}$ and $\mathrm{C}_{4} \mathrm{H}$ are positively correlated indicating that these two classes of molecules can coexist during the embedded protostellar stage. The $\mathrm{C}_{4} \mathrm{H} / \mathrm{CH}_{3} \mathrm{OH}$ gas abundance ratio tentatively correlates with the $\mathrm{CH}_{4} / \mathrm{CH}_{3} \mathrm{OH}$ ice abundance ratio in the same lines of sight. This relationship supports a scenario where carbon chain formation in protostellar envelopes begins with $\mathrm{CH}_{4}$ ice desorption.

Keywords: Astrochemistry - ISM: molecules - stars: formation - stars: protostars
\end{abstract}

\section{INTRODUCTION}

Large organic molecules have been widely observed across different stages of star-formation (Herbst \& van Dishoeck 2009; Sakai \& Yamamoto 2013). Their chemistry is of great interest because large interstellar molecules may serve as precursors to prebiotic chemistry on nascent planets. Large organic molecules also have a great potential as molecular probes. In general, molecular abundance patterns contain information about the past and current environment where they reside. This relationship can be exploited to constrain interstellar environments using molecular line observations, if the chemistry of the target molecule is well understood. Larger molecules present numerous rotational lines at millimeter and centimeter wavelengths and are therefore especially appropriate from an excitation point of view. They suffer, however, from an often poorly constrained formation chemistry.

In this paper we explore how the relationship of two kinds of larger organic molecules constrain their chemistry. Interstellar organics are classified as either saturated organic molecules or unsaturated carbon chains. When saturated organic molecules reach sizes of 6 atoms or greater, they are known as complex organic molecules (COMs). These two classes of molecules have been generally supposed to form and exist in very different interstellar and circumstellar environments (Herbst \& van Dishoeck 2009).

Carbon chain molecules were first observed in the cold, dark cloud TMC-1 in the form of cyanopolyynes

\footnotetext{
dgraninger@cfa.harvard.edu

${ }^{1}$ Departments of Chemistry and Mathematics, Dickinson College, Carlisle, PA, 17013
}

(Little et al. 1977; Kroto et al. 1978; Broten et al. 1978). In these environments, carbon chains form through efficient low-temperature ion-molecule reactions in the gas phase (Herbst \& Leung 1989; Ohishi \& Kaifu 1998). In 2008, Sakai et al. detected the first evidence of carbon chains in an unexpected region - the warm, inner regions of a low-mass protostar. Detections toward a second source (Sakai et al. 2009a) suggested that carbon chains are common toward some classes of protostars. The term "warm carbon chain chemistry" (WCCC) was coined to describe this type of source. Based on low-temperature modeling, ion-molecule chemistry and chemical inheritance are not sufficient to produce the high abundances of carbon chains observed in WCCC sources. Sakai et al. (2008) proposed that additional carbon chains can be formed in protostellar envelopes when methane $\left(\mathrm{CH}_{4}\right)$, a common interstellar ice, sublimates at $25 \mathrm{~K}$ Oberg et al. 2008). In the gas phase, $\mathrm{CH}_{4}$ reacts with $\mathrm{C}^{+}$to efficiently form carbon chains. This theory has been validated by chemical models (Aikawa et al. 2012; Hassel et al. 2011).

COMs were initially observed in hot cores present toward high-mass protostars (Blake et al. 1987). During the past two decades it has become clear, however, that COMs are abundant in many other circumstellar and interstellar environments as well (see e.g. Cazaux et al. 2003; Arce et al. 2008). COMs have even been detected toward cold cloud cores, the traditional site of formation for carbon chains, (Oberg et al. 2010; Bacmann et al. 2012; Cernicharo et al. 2012). Based on experiments and models, COMs form efficiently on grain surfaces through energetic processing of simple ices and are released into the gas phase via thermal or non-thermal processes (Garrod \& Herbst 2006; Garrod et al. 2008; 
Oberg et al. 2009b). Recent theoretical work has shown that COMs may also be formed in the gas-phase following the desorption of methanol $\left(\mathrm{CH}_{3} \mathrm{OH}\right)$ (Balucani et al. 2015).

Both models and observations thus reveal that both carbon chains and complex organics can form at a range of temperatures and may therefore coexist in some sources. Based on a small source sample (2 WCCC sources and 3 hot corinos), Sakai et al. (2008, 2009a) found that carbon chains and COMs are not detected in large abundances in the same low-mass protostellar sources, however. They suggest that this anti-correlation stems from differences in star formation time scales, which results in different compositions of the ice grains in the protostellar envelope, for WCCC vs. hot corino sources. If the starless core phase is short, the bulk of the carbonaceous ice form from accretion of $\mathrm{C}$ atoms. The carbon atoms are hydrogenated to form $\mathrm{CH}_{4}$ and the release of $\mathrm{CH}_{4}$ during protostellar formation promotes warm carbon chain chemistry. If the starless core is longlived, most of the carbon atoms in the gas phase have had time to react and form $\mathrm{CO}$, resulting in a $\mathrm{CO}$ dominated ice. The formation of $\mathrm{CH}_{3} \mathrm{OH}$ is through $\mathrm{CO}$ hydrogenation and $\mathrm{CH}_{3} \mathrm{OH}$ is the starting point of COM chemistry on grains (Sakai \& Yamamoto 2013).

In this study, we explore the relationship between the COM precursor $\mathrm{CH}_{3} \mathrm{OH}$ and prototypical carbon chain $\mathrm{C}_{4} \mathrm{H}$ in a sample of embedded protostars, selected from the Spitzer $c 2 d$ (cores to disk) ice survey. We further explore the relationship between $\mathrm{C}_{4} \mathrm{H}$ and $\mathrm{CH}_{3} \mathrm{OH}$, and ice abundances in the same lines of sight. In $\S 2$, we describe our source sample and selection criteria. The IRAM $30 \mathrm{~m}$ observations are described in $\S 3$. The results and discussion are in $\S 4$ and $\S 5$ and the conclusions can be found in $\oint 6$.

\section{SAMPLE SELECTION}

Our sources were selected from the Spitzer $c 2 d$ ice sample (Evans et al. 2003; Boogert et al. 2008). From the 51 sources in the Spitzer $c 2 d$ ice sample presented in Boogert et al. (2008), we initially constrained ourselves to the northern low-mass sources as they are easily observable with the IRAM $30 \mathrm{~m}$, leaving us with 26 sources. The sources are characterized into different classes based on their spectral energy distributions, and in particular their IR spectral indices, $\alpha_{\mathrm{IR}}$, defined as the slope between 2 and $24 \mu \mathrm{m}$. We chose sources with $\alpha_{\mathrm{IR}}>0.3$, which defines class 0/I sources (Wilking et al. 2001). These sources are often, but not always, associated with young, embedded YSOs; this cut left us with 19 sources. We then removed B1-b, IRAS 03301+3111, and EC 92 from our sample. B1-b was removed as previous observations of this source exist, IRAS 03301+3111 was removed as the upper limits on the ice abundances did not place any strong constraints on the data, and EC 92 was removed because it was not resolvable from SVS 4-5 within the beam of the IRAM $30 \mathrm{~m}$.

Table 1 lists the source coordinates, bolometric luminosities, envelope masses and the IR SED indices, together with the $\mathrm{H}_{2} \mathrm{O}, \mathrm{CH}_{4}$, and $\mathrm{CH}_{3} \mathrm{OH}$ ice abundances of our final sample. Our sources span $\alpha_{\text {IR }}$ from 0.34 - 2.70 and are situated in the Perseus, Taurus, Serpens, L1014, and CB244 clouds. The envelope masses are between $0.1-17.7 \mathrm{M}_{\odot}$, and bolometric luminosi- ties are between $0.32-38 \mathrm{~L}_{\odot}$. Previous observations of WCCC sources fall within our range of bolometric luminosities (Andre et al. 2000; Chen et al. 1997). The $\mathrm{H}_{2} \mathrm{O}$ ice column is between $0.4-39 \times 10^{18} \mathrm{~cm}^{-2}$ and the $N_{\mathrm{CH}_{3} \mathrm{OH}} / N_{\mathrm{H}_{2} \mathrm{O}}$ and $N_{\mathrm{CH}_{4}} / N_{\mathrm{H}_{2} \mathrm{O}}$ are $2-25 \%$ and $1.6-11 \%$, respectively. Six of our sources (B1-a, B5 IRS 1, L1489 IRS, IRAS 04108+2803, SVS 4-5, and IRAS $03235+3004$ ) were part of a study by Oberg et al. (2014) on COMs, including $\mathrm{CH}_{3} \mathrm{OH}$, in low-mass protostars. For these sources, we recalculate the $\mathrm{CH}_{3} \mathrm{OH}$ column densities using the $\mathrm{CH}_{3} \mathrm{OH}$ integrated line intensities from Oberg et al. (2014) and our rotation diagram method.

\section{OBSERVATIONS}

All sources were observed with the IRAM 30m telescope using the EMIR $90 \mathrm{GHz}$ receiver and the Fourier Transform Spectrometer (FTS) backend. Six of the sources (B1-a, B5 IRS 1, L1489 IRS, IRAS 04108+2803, SVS 4-5, and IRAS 03235+3004) were observed on June $12-16,2013$ at $93-101 \mathrm{GHz}$ and $109-117 \mathrm{GHz}$. The remaining sources were observed on July 23 - 28, 2014 at $92-100 \mathrm{GHz}$ and $108-116 \mathrm{GHz}$. The spectral resolution for both sets of observations was $200 \mathrm{kHz}$ and the sideband rejection was $-15 \mathrm{~dB}$ (Carter et al. 2012).

The pointing accuracy was checked every $1-2 \mathrm{hrs}$ and found to be within $2^{\prime \prime}-3^{\prime \prime}$. Focus, which was checked every $4 \mathrm{hrs}$, remained stable with corrections of $<0.4 \mathrm{~mm}$. For the data set obtained in June 2013, both the position switching and wobbler switching modes were used during observations, but the position switching spectra has been excluded from this paper because of severe baseline instabilities; only wobbler switching was used for the July 2014 observations with a wobbler throw of $2^{\prime}$. We exclude IRAS $03254+3050$ from further analysis due to significant self-absorption in the molecular targets of this study. No other source displayed signs of self-absorption in $\mathrm{CH}_{3} \mathrm{OH}$ or $\mathrm{C}_{4} \mathrm{H}$. We also inspected the shapes of stronger lines, e.g. CN, in individual integrations and found no self-absorption in any high-density tracing line. This indicates a lack of dense material in the off-position and therefore a low probability of $\mathrm{CH}_{3} \mathrm{OH}$ and $\mathrm{C}_{4} \mathrm{H}$-emitting, material in the wobble off-positions.

The spectra were reduced using CLASS with a global baseline fit to each $4 \mathrm{GHz}$ spectral chunk using four to seven line-free windows. Individual scans were baseline subtracted and averaged, and antenna temperature, $T_{a}^{*}$, was converted to main beam temperature, $T_{m b}$, by applying forward and beam efficiency values of 0.95 and 0.81. Using literature source velocities, the spectra were converted to rest frequency with additional adjustments made based on the $\mathrm{CH}_{3} \mathrm{OH} 2-1$ ladder. The $\mathrm{CH}_{3} \mathrm{OH}$ 2-1 ladder emission was compared with previous observations for a subset of sources and was found to agree within 10\% (Oberg et al. 2009a).

\section{RESULTS}

$\mathrm{CH}_{3} \mathrm{OH}$ is detected in all 15 analyzed sources and $\mathrm{C}_{4} \mathrm{H}$ in 13 sources at greater than $3 \sigma$. Two $\mathrm{C}_{4} \mathrm{H}$ upper limits are also reported. Figures 1 and 2 display the $\mathrm{CH}_{3} \mathrm{OH}$ and $\mathrm{C}_{4} \mathrm{H}$ lines toward all observed sources. For ease of

\footnotetext{
$\dagger$ http://www.iram.fr/IRAMFR/GILDAS
} 
Table 1

Source information of the complete 16-object $c 2 d$ embedded protostar sample with ice detections

\begin{tabular}{|c|c|c|c|c|c|c|c|c|c|}
\hline Source & $\begin{array}{c}\text { R.A. } \\
\text { (J2000.0) }\end{array}$ & $\begin{array}{c}\text { Dec } \\
(\mathrm{J} 2000.0)\end{array}$ & Cloud & $\begin{array}{c}\mathrm{L}_{\text {bol }} \\
\mathrm{L}_{\odot}\end{array}$ & $\begin{array}{c}\mathrm{M}_{\text {env }} \\
\mathrm{M}_{\odot}\end{array}$ & $\alpha_{\mathrm{IR}}^{\mathrm{a}}$ & $\begin{array}{c}N\left(\mathrm{H}_{2} \mathrm{O}\right)^{\mathrm{a}} \\
10^{18} \mathrm{~cm}^{-2}\end{array}$ & $\begin{array}{c}\mathrm{X}_{\mathrm{CH}_{3} \mathrm{OH}}^{\mathrm{b}} \\
\% \mathrm{H}_{2} \mathrm{O}\end{array}$ & $\begin{array}{l}\mathrm{X}_{\mathrm{CH}_{4}}^{\mathrm{c}} \\
\% \mathrm{H}_{2} \mathrm{O}\end{array}$ \\
\hline $\mathrm{B} 1-\mathrm{a}^{\mathrm{d}}$ & 03:33:16.67 & $31: 07: 55.1$ & Perseus & $1.3^{\mathrm{e}}$ & $2.8^{\mathrm{e}}$ & 1.87 & $10.39[2.26]$ & $<1.9$ & $<5.7$ \\
\hline SVS $4-5^{\mathrm{d}}$ & $18: 29: 57.59$ & 01:13:00.6 & Serpens & $38^{\mathrm{f}}$ & - & 1.26 & $5.65[1.13]$ & $25.2[3.5]$ & $6.1[1.7]$ \\
\hline B1-c & 03:33:17.89 & $31: 09: 31.0$ & Perseus & $3.7^{\mathrm{e}}$ & $17.7^{\mathrm{e}}$ & 2.66 & $29.55[5.65]$ & $<7.1$ & $5.4[1.4]$ \\
\hline IRAS $23238+7401$ & $23: 25: 46.65$ & $74: 17: 37.2$ & CB244 & - & - & 0.95 & $12.95[2.26]$ & $<3.6$ & $<7.4$ \\
\hline L1455 IRS3 & 03:28:00.41 & 30:08:01.2 & Perseus & $0.32^{\mathrm{e}}$ & $0.2^{\mathrm{g}}$ & 0.98 & $0.92[0.37]$ & $<12.5$ & - \\
\hline B5 IRS $1^{\mathrm{d}}$ & $03: 47: 41.61$ & $32: 51: 43.8$ & Perseus & $4.7^{\mathrm{e}}$ & $4.2^{\mathrm{e}}$ & 0.78 & $2.26[0.28]$ & $<3.7$ & - \\
\hline L1455 SMM1 & $03: 27: 43.25$ & $30: 12: 28.8$ & Perseus & $3.1^{\mathrm{e}}$ & $5.3^{\mathrm{e}}$ & 2.41 & $18.21[2.82]$ & $<13.5$ & $5.8[0.9]$ \\
\hline IRAS $03245+3002$ & 03:27:39.03 & $30: 12: 59.3$ & Perseus & $7.0^{\mathrm{e}}$ & $5.3^{\mathrm{e}}$ & 2.70 & $39.31[5.65]$ & $<9.8$ & $1.7[0.3]$ \\
\hline L1014 IRS & $21: 24: 07.51$ & 49:59:09.0 & L1014 & - & - & 1.28 & $7.16[0.91]$ & $3.1[0.8]$ & $7.1[2.3]$ \\
\hline IRAS $04108+2803^{\mathrm{d}}$ & 04:13:54.72 & $28: 11: 32.9$ & Taurus & $0.62^{\mathrm{h}}$ & - & 0.90 & $2.87[0.4]$ & $<3.5$ & $<11$ \\
\hline IRAS $03235+3004^{\mathrm{d}}$ & $03: 26: 37.45$ & $30: 15: 27.9$ & Perseus & $1.9^{\mathrm{e}}$ & $2.4^{\mathrm{e}}$ & 1.44 & $14.48[2.26]$ & $4.2[1.2]$ & $4.3[1.4]$ \\
\hline L1489 IRS ${ }^{\mathrm{d}}$ & 04:04:43.07 & $26: 18: 56.4$ & Taurus & $3.7^{\mathrm{h}}$ & $0.1^{\mathrm{i}}$ & 1.10 & $4.26[0.51]$ & $4.9[1.5]$ & $3.1[0.2]$ \\
\hline HH 300 & $04: 26: 56.30$ & $24: 43: 35.3$ & Taurus & $1.27^{\mathrm{h}}$ & $0.03^{\mathrm{j}}$ & 0.79 & $2.59[0.25]$ & $<6.7$ & $<14$ \\
\hline IRAS $03271+3013$ & 03:30:15.16 & $30: 23: 48.8$ & Perseus & $0.8^{\mathrm{e}}$ & $1.2^{\mathrm{e}}$ & 2.06 & $7.69[1.76]$ & $<5.6$ & $<1.6$ \\
\hline L1448 IRS1 & 03:25:09.44 & $30: 46: 21.7$ & Perseus & $17.0^{\mathrm{e}}$ & $16.3^{\mathrm{e}}$ & 0.34 & $0.47[0.16]$ & $<14.9$ & - \\
\hline IRAS $03254+3050$ & 03:28:34.51 & 31:00:51.2 & Perseus & - & $0.3^{\mathrm{e}}$ & 0.90 & $3.66[0.47]$ & $<4.6$ & 4.0 \\
\hline
\end{tabular}

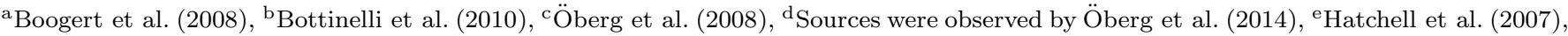
f Pontoppidan et al. (2004), ' Enoch et al. (2009), '
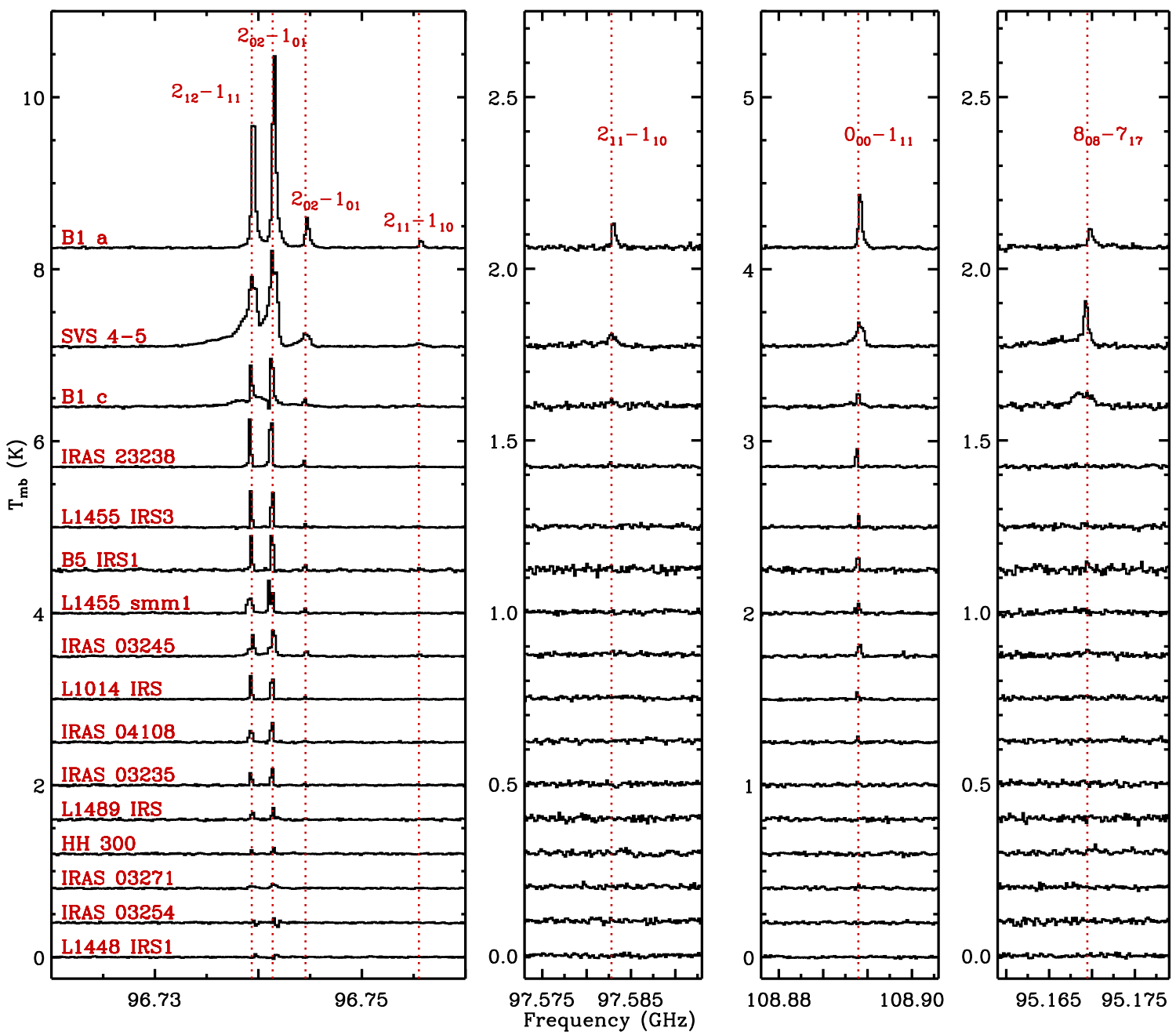

Figure 1. IRAM 30m observations of the $\mathrm{CH}_{3} \mathrm{OH}$ lines detected toward the low-mass YSO sample. The spectra have been shifted with the systemic velocity of each source. 

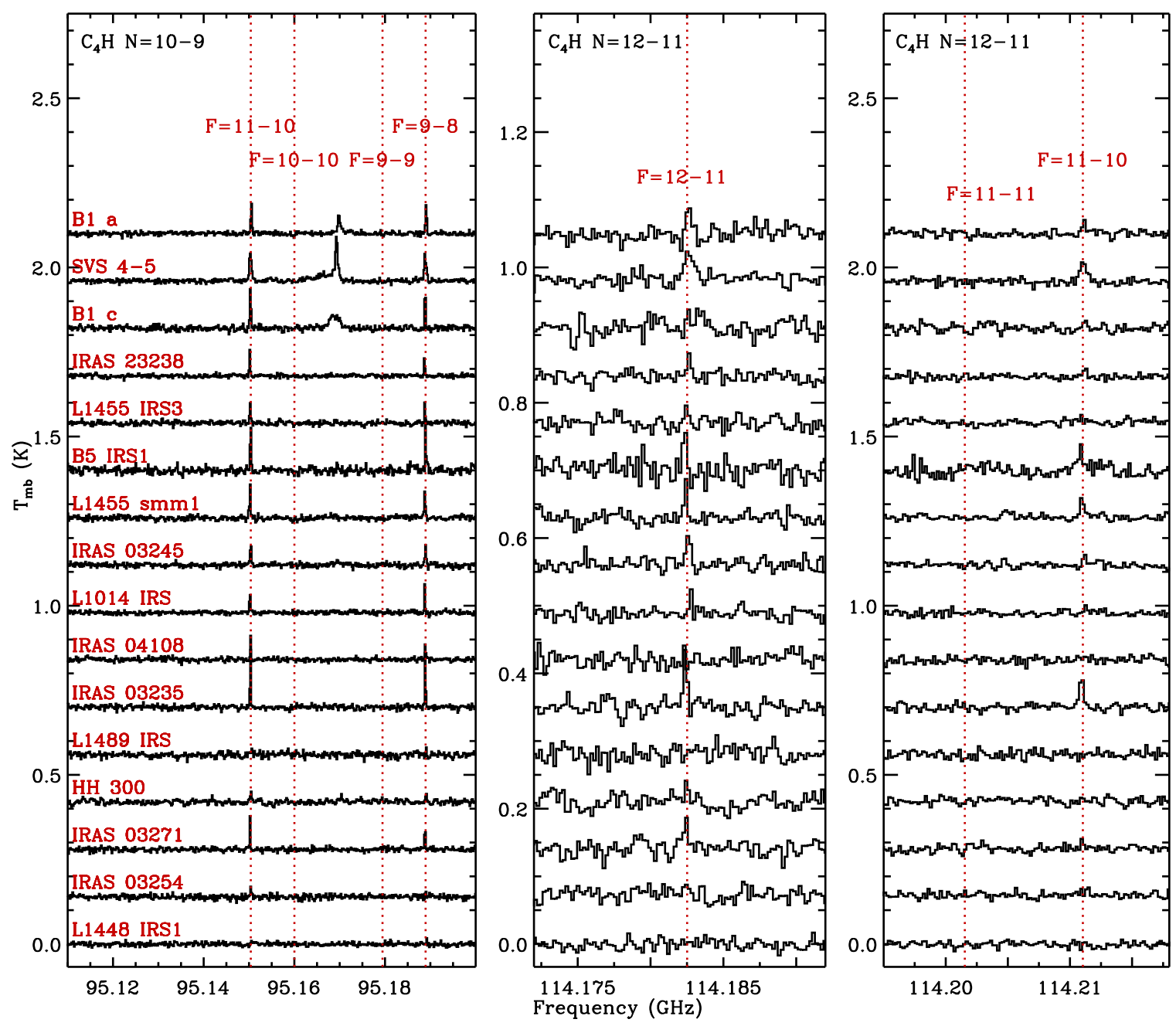

Figure 2. IRAM $30 \mathrm{~m}$ observations of the $\mathrm{C}_{4} \mathrm{H}$ lines detected toward the low-mass YSO sample. The spectra have been shifted with the systemic velocity of each source. 


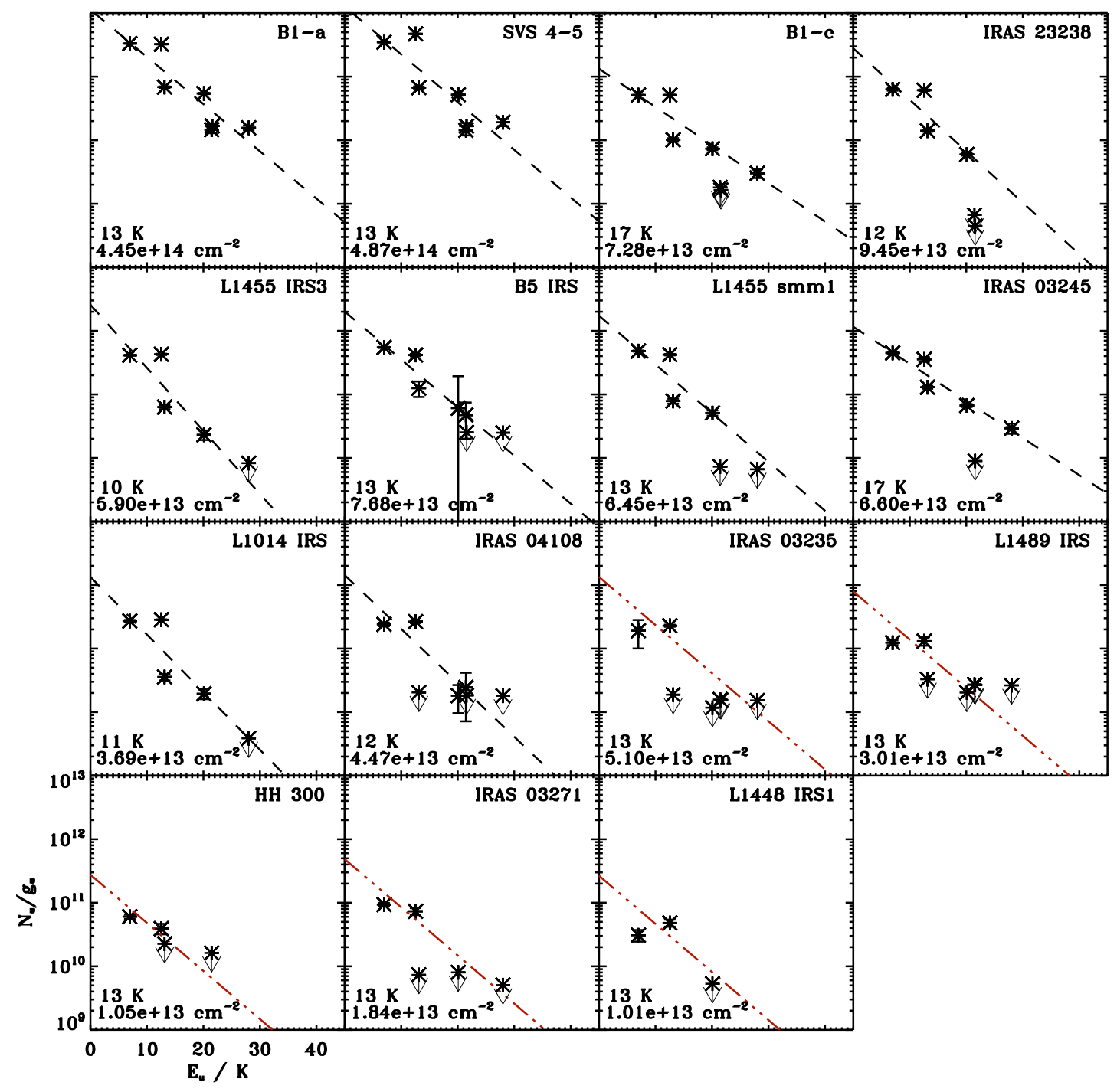

Figure 3. Rotation Diagrams for $\mathrm{CH}_{3} \mathrm{OH}$. The black dashed lines represent the fit to the lines. When a line could not be fit, denoted by the red dot-dash line, the average rotational temperature was used to derive the column density. Where no error bars are seen, the errors bars are smaller than the symbols.

comparison, the $\mathrm{CH}_{3} \mathrm{OH}$ lines in Figure 1 are ordered in decreasing $\mathrm{CH}_{3} \mathrm{OH}$ brightness from the top. Figure 2 is ordered identically. Tables 2 and 3 list the integrated intensity of the $\mathrm{CH}_{3} \mathrm{OH}$ and $\mathrm{C}_{4} \mathrm{H}$ lines, respectively. The integrated intensity of $\mathrm{CH}_{3} \mathrm{OH}$ lines of sources studied by Oberg et al. (2014) can be found in Table 2 of that paper. Most $\mathrm{CH}_{3} \mathrm{OH}$ and all $\mathrm{C}_{4} \mathrm{H}$ lines could be fit by a single Gaussian. Toward SVS 4-5 and B1-c, the $\mathrm{CH}_{3} \mathrm{OH}$ lines display substantial wings, probably due to outflows in the beam, and these features were fit with 2-3 Gaussian components. Only the narrow central Gaussian components were used to derived the listed column densities, since their properties agreed the best with the $\mathrm{C}_{4} \mathrm{H}$ line characteristics in the same lines of sight.

To determine the column densities of $\mathrm{CH}_{3} \mathrm{OH}$ and $\mathrm{C}_{4} \mathrm{H}$, we used the rotation diagram method of Goldsmith \& Langer (1999) and assumed optically thin lines and local thermodynamic equilibrium (LTE) at a single temperature. Figures 3 and 4 display the rotation diagrams obtained for $\mathrm{CH}_{3} \mathrm{OH}$ and $\mathrm{C}_{4} \mathrm{H}$, respectively. Sources observed by Oberg et al. (2014) were refit using a single-component rotation diagram to be consistent with the column density determinations of $\mathrm{C}_{4} \mathrm{H}$ and the new $\mathrm{CH}_{3} \mathrm{OH}$ observations.

Table 4 lists the derived column densities and rotational temperature obtained from the rotation diagram analysis. In situations where there were not enough lines with $\mathrm{SNR}>3 \sigma$, the rotational temperature is assumed to be the average of the observed rotational temperatures; in these cases, $\mathrm{T}_{\text {rot }}$ is italicized. On average, $\mathrm{T}_{\text {rot }}$ for $\mathrm{CH}_{3} \mathrm{OH}$ is $13 \pm 2 \mathrm{~K}$ and $24 \pm 5 \mathrm{~K}$ for $\mathrm{C}_{4} \mathrm{H}$. It is important to note that for this class of objects the $\mathrm{CH}_{3} \mathrm{OH}$ rotational temperatures do not necessarily reflect the kinetic temperatures, but are rather lower limits (Oberg et al. 2014). The difference in excitation temperature for $\mathrm{CH}_{3} \mathrm{OH}$ and $\mathrm{C}_{4} \mathrm{H}$ can thus not be used to constrain the relative emission regions of $\mathrm{CH}_{3} \mathrm{OH}$ and $\mathrm{C}_{4} \mathrm{H}$. 


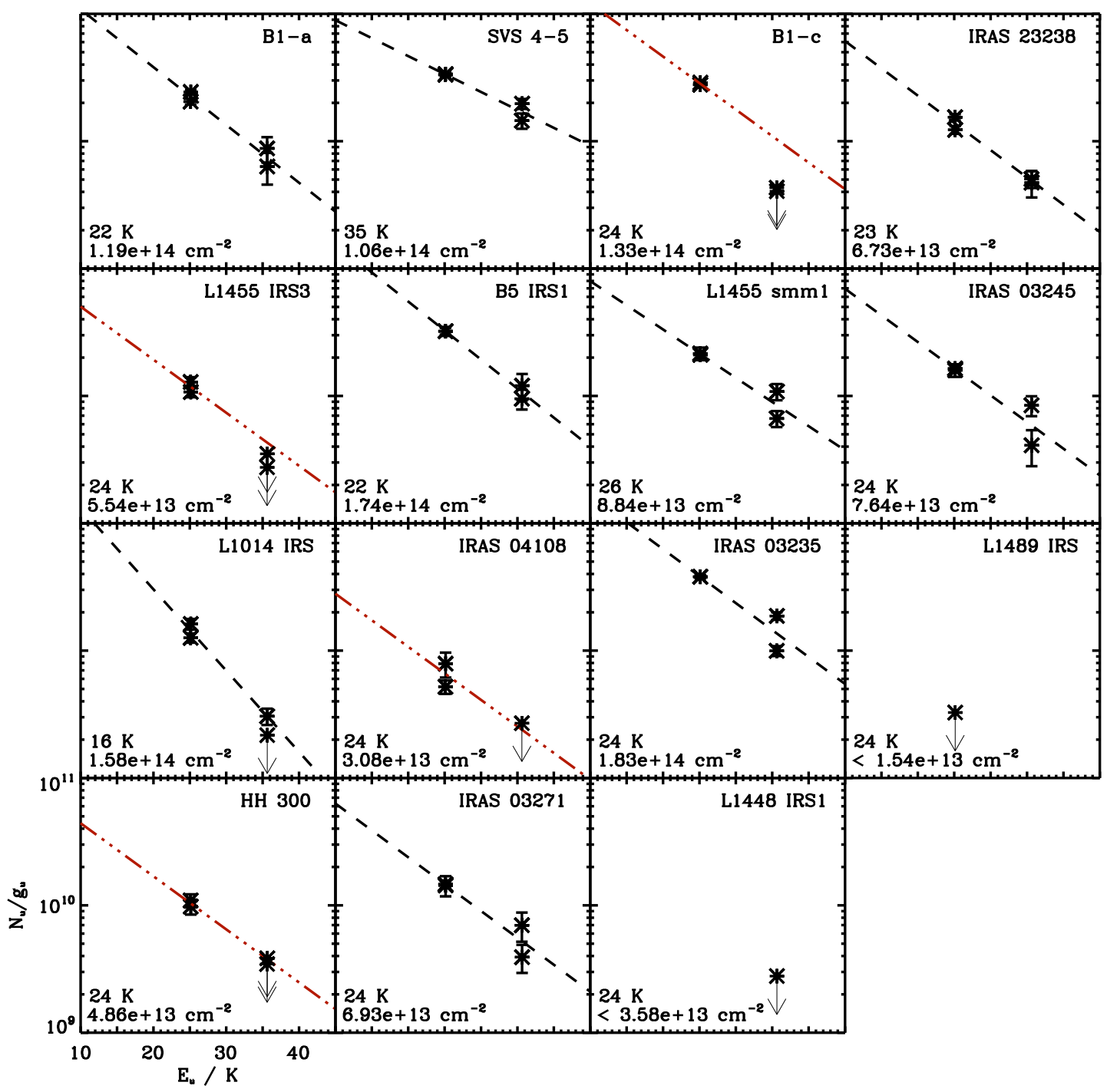

Figure 4. Rotation Diagrams for $\mathrm{C}_{4} \mathrm{H}$. The black dashed lines represent the fit to the lines. When a line could not be fit, denoted by the red dot-dash line, the average rotational temperature was used to derive the column density. Where no error bars are seen, the errors bars are smaller than the symbols.

Table 2

Integrated $\mathrm{CH}_{3} \mathrm{OH}$ Line Intensities in $\mathrm{K} \mathrm{km} \mathrm{s}^{-1}$

\begin{tabular}{lccccccc}
\hline \hline & $95.914 \mathrm{GHz}$ & $96.739 \mathrm{GHz}$ & $96.741 \mathrm{GHz}$ & $96.745 \mathrm{GHz}$ & $96.756 \mathrm{GHz}$ & $97.583 \mathrm{GHz}$ & $108.894 \mathrm{GHz}$ \\
Source & $212-1_{11}$ & $2_{12}-1_{11}$ & $2_{02}-1_{01}$ & $2_{02}-1_{01}$ & $2_{11}-1_{10}$ & $211-1_{10}$ & $000-111$ \\
\hline B1-c & $<0.013$ & $0.361[0.004]$ & $0.481[0.004]$ & $0.069[0.004]$ & $0.022[0.003]$ & $<0.012$ & $0.065[0.004]$ \\
IRAS 23238+7401 & $<0.005$ & $0.430[0.011]$ & $0.591[0.027]$ & $0.056[0.002]$ & - & $<0.003$ & $0.090[0.003]$ \\
L1455 IRS3 & - & $0.300[0.007]$ & $0.387[0.005]$ & $0.022[0.004]$ & $<0.006$ & - & $0.040[0.003]$ \\
L1455 SMM1 & $<0.005$ & $0.297[0.008]$ & $0.452[0.009]$ & $0.048[0.004]$ & $<0.005$ & - & $0.050[0.004]$ \\
IRAS 03245+3002 & - & $0.250[0.021]$ & $0.421[0.015]$ & $0.063[0.003]$ & $0.021[0.004]$ & $<0.006$ & $0.083[0.004]$ \\
L1014 IRS & - & $0.200[0.002]$ & $0.254[0.010]$ & $0.018[0.003]$ & $<0.003$ & - & $0.023[0.003]$ \\
HH 300 & $<0.011$ & $0.027[0.005]$ & $0.057[0.005]$ & - & - & - & $<0.014$ \\
IRAS 03271+3013 & - & $0.051[0.007]$ & $0.088[0.005]$ & $<0.008$ & $<0.004$ & - & $<0.005$ \\
L1448 IRS1 & - & $0.034[0.004]$ & $0.029[0.006]$ & $<0.005$ & - & - & - \\
\hline \hline
\end{tabular}


Table 3

Integrated $\mathrm{C}_{4} \mathrm{H}$ Line Intensities in $\mathrm{K} \mathrm{km} \mathrm{s}^{-1}$

\begin{tabular}{lcccc}
\hline \hline & $95.150 \mathrm{GHz}$ & $95.189 \mathrm{GHz}$ & $114.183 \mathrm{GHz}$ & $114.221 \mathrm{GHz}$ \\
Source & $\mathrm{N}=10-9, \mathrm{~F}=11-10$ & $\mathrm{~N}=10-9, \mathrm{~F}=9-8$ & $\mathrm{~N}=12-11, \mathrm{~F}=12-11$ & $\mathrm{~N}=12-11, \mathrm{~F}=11-10$ \\
\hline B1-a & $0.088[0.005]$ & $0.094[0.004]$ & $0.054[0.012]$ & $0.036[0.010]$ \\
SVS 4-5 & $0.142[0.007]$ & $0.130[0.008]$ & $0.090[0.013]$ & $0.112[0.010]$ \\
B1-c & $0.119[0.008]$ & $0.111[0.004]$ & $<0.027$ & $<0.023$ \\
IRAS 23238+7401 & $0.066[0.004]$ & $0.047[0.003]$ & $0.031[0.005]$ & $0.027[0.006]$ \\
L1455 IRS3 & $0.055[0.005]$ & $0.041[0.004]$ & $<0.017$ & $<0.019$ \\
B5 IRS 1 & $0.138[0.008]$ & $0.124[0.010]$ & $0.059[0.010]$ & $0.068[0.016]$ \\
L1455 SMM1 & $0.092[0.010]$ & $0.081[0.005]$ & $0.041[0.006]$ & $0.061[0.009]$ \\
IRAS 03245+3002 & $0.070[0.005]$ & $0.061[0.007]$ & $0.052[0.009]$ & $0.023[0.007]$ \\
L1014 IRS & $0.054[0.005]$ & $0.062[0.006]$ & $0.19[0.003]$ & $<0.012$ \\
IRAS 04108+2803 & $0.022[0.003]$ & $0.030[0.007]$ & $<0.017$ & - \\
IRAS 03235+3004 & $0.163[0.003]$ & $0.147[0.004]$ & $0.062[0.005]$ & $0.106[0.007]$ \\
L1489 IRS & - & $<0.013$ & - & - \\
HH 300 & $0.047[0.006]$ & $0.038[0.005]$ & $<0.021$ & $<0.022$ \\
IRAS 03271+3013 & $0.062[0.004]$ & $0.055[0.010]$ & $0.043[0.011]$ & $0.022[0.006]$ \\
L1448 IRS1 & - & - & - & $<0.016$ \\
\hline \hline
\end{tabular}

Table 4

Column Densities and Rotational Temperatures for $\mathrm{CH}_{3} \mathrm{OH}$ and $\mathrm{C}_{4} \mathrm{H}$

\begin{tabular}{|c|c|c|c|c|}
\hline Source & $\begin{array}{c}\mathrm{N}\left(\mathrm{CH}_{3} \mathrm{OH}\right) \\
10^{13} \mathrm{~cm}^{-2}\end{array}$ & $\begin{array}{c}\mathrm{T}_{\text {rot }}\left(\mathrm{CH}_{3} \mathrm{OH}\right)^{\mathrm{a}} \\
\mathrm{K}\end{array}$ & $\begin{array}{c}\mathrm{N}\left(\mathrm{C}_{4} \mathrm{H}\right) \\
10^{13} \mathrm{~cm}^{-2}\end{array}$ & $\begin{array}{c}\mathrm{T}_{\text {rot }}\left(\mathrm{C}_{4} \mathrm{H}\right)^{\mathrm{a}} \\
\mathrm{K}\end{array}$ \\
\hline $\mathrm{B} 1-\mathrm{a}^{\mathrm{b}}$ & $44[14]$ & $13[3]$ & $11.9[2.8]$ & $22[4]$ \\
\hline SVS $4-5^{b}$ & $49[19]$ & $13[4]$ & $10.6[2.1]$ & $35[8]$ \\
\hline B1-c & $7.3[2.2]$ & $17[5]$ & $13.3[0.7]$ & $24[5]$ \\
\hline IRAS $23238+7401$ & $9.4[4.4]$ & $12[5]$ & $6.7[1.0]$ & $23[3]$ \\
\hline L1455 IRS3 & $5.9[3.6]$ & $10[4]$ & $5.5[0.5]$ & $24[5]$ \\
\hline B5 IRS $1^{\mathrm{b}}$ & $7.7[2.0]$ & $13[6]$ & $17.4[2.6]$ & $22[2]$ \\
\hline L1455 SMM1 & $6.4[3.3]$ & $13[6]$ & $8.8[2.8]$ & $26[7]$ \\
\hline IRAS $03245+3002$ & $6.6[1.2]$ & $17[3]$ & $7.6[3.5]$ & $24[9]$ \\
\hline L1014 IRS & $3.7[2.4]$ & $11[6]$ & $15.8[4.2]$ & $16[2]$ \\
\hline IRAS $04108+2803^{\mathrm{b}}$ & $4.5[1.9]$ & $12[4]$ & $3.1[0.4]$ & $24[5]$ \\
\hline IRAS $03235+3004^{\mathrm{b}}$ & $5.1[0.3]$ & $13[2]$ & $18.3[7.4]$ & $24[7]$ \\
\hline L1489 IRS ${ }^{\mathrm{b}}$ & $3.0[0.6]$ & 13 [2] & $<1.5$ & $24[5]$ \\
\hline HН 300 & $1.0[0.4]$ & 13 [2] & $4.9[0.6]$ & $24[5]$ \\
\hline IRAS $03271+3013$ & $1.8[0.2]$ & 13 [2] & $6.9[2.5]$ & $27[7]$ \\
\hline L1448 IRS1 & $1.0[0.2]$ & $13[2]$ & $<3.6$ & $24[5]$ \\
\hline
\end{tabular}

${ }^{\mathrm{a}} \mathrm{T}_{\mathrm{rot}}$ values in italics are assumed rotational temperatures and are based on the average $\mathrm{T}_{\text {rot }}$ in the source with standard deviation errors. The listed errors are the $1 \sigma$ uncertainty. ${ }^{b}$ Sources observed by Öberg et al. (2014) 


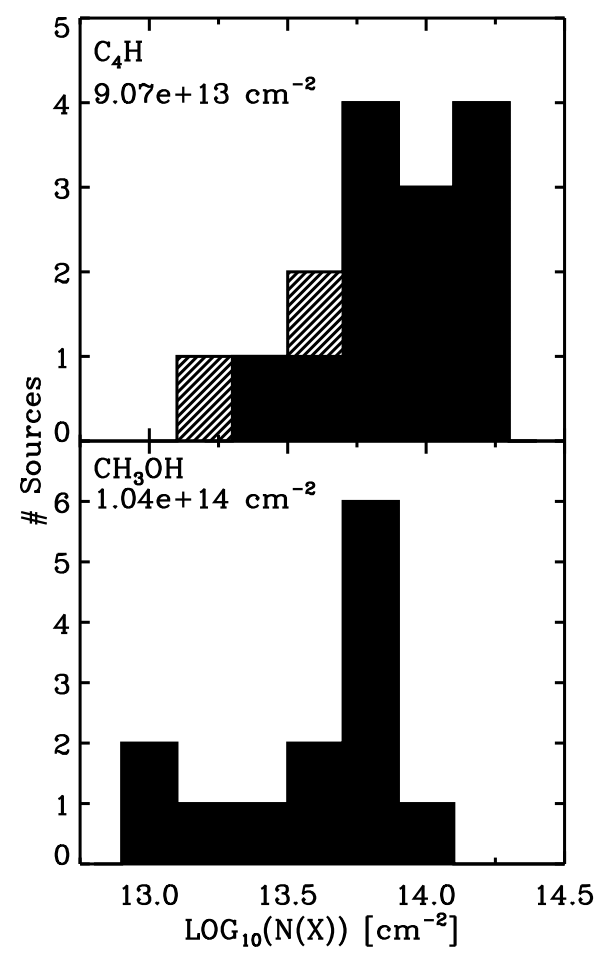

Figure 5. Histograms of the column density of $\mathrm{C}_{4} \mathrm{H}$ and $\mathrm{CH}_{3} \mathrm{OH}$. The solid bars are detections and line-filled bars indicate upper limits. The average column density is also listed in the upper left hand corner.

The observed column density distributions of $\mathrm{C}_{4} \mathrm{H}$ and $\mathrm{CH}_{3} \mathrm{OH}$ are displayed in Figure 5 along with their respective average column densities. The column densities of both species span approximately an order of magnitude and the average column densities of $9.1 \times 10^{13}$ $\mathrm{cm}^{-2}$ and $1.0 \times 10^{14} \mathrm{~cm}^{-2}$ for $\mathrm{C}_{4} \mathrm{H}$ and $\mathrm{CH}_{3} \mathrm{OH}$, respectively are similar. The WCCC sources from Sakai et al. (2008, 2009a, b) overlap with the high end of our $\mathrm{C}_{4} \mathrm{H}$ distribution. Classical hot corino sources (Maret et al. 2005; Sakai et al. 2009a overlap with the high-end of the $\mathrm{CH}_{3} \mathrm{OH}$ distribution and the low end of our distribution for $\mathrm{C}_{4} \mathrm{H}$. However, as is seen below, classical hot corino sources form a distinct sub-group when simultaneously taking into account the column densities of both species, compared to any sources in our sample.

Figure 6 displays the column density correlation plots for $\mathrm{CH}_{3} \mathrm{OH}$ and $\mathrm{C}_{4} \mathrm{H}$, both absolute and normalized to the $\mathrm{H}_{2} \mathrm{O}$ ice column. As $\mathrm{H}_{2} \mathrm{O}$ ice is the first ice to form and the last to desorb during star-formation, its column should be a good proxy of the envelope material in the line of sight (Boogert et al. 2015).

For both column density correlation plots, there appears to be a positive correlation. To determine the statistical significance of the correlation, a Spearman's (rho) rank correlation tests were performed. The absolute column densities are not significantly correlated. By contrast, the normalized (to water ice) column densities are significantly positively correlated at the $>99 \%$ level applying the Spearman's rank correlation test.

In Figure 6, the warm carbon chain source, L1527, is displayed as a teal square and falls within our observed columns for both $\mathrm{CH}_{3} \mathrm{OH}$ and $\mathrm{C}_{4} \mathrm{H}$ (Sakai et al. 2008, 2009b). The red diamonds display three classic hot corino sources (Maret et al. 2005; Sakai et al. 2009b). The three hot corino sources and the WCCC source L1527 in Figure 6] display an anti-correlation. This is consistent with the anti-correlation noted by Sakai et al. (2008). When combing the hot corino and WCCC sources with our sample, we see two distinct regions: a positive correlation for low to medium column densities of $\mathrm{CH}_{3} \mathrm{OH}$, while the hot corino sources occupy a unique space characterized by high $\mathrm{CH}_{3} \mathrm{OH}$ and low $\mathrm{C}_{4} \mathrm{H}$ column densities.

The difference between our sample and the hot corino sources suggest that $\mathrm{CH}_{3} \mathrm{OH} / \mathrm{C}_{4} \mathrm{H}$ may trace an evolutionary sequence. We used the IR spectral indices, $\alpha_{\text {IR }}$, as a proxy for age since $\alpha_{\text {IR }}$ decreasing signifies an increasing age (Wilking et al. 2001). We find no relationship for the $\mathrm{CH}_{3} \mathrm{OH} / \mathrm{C}_{4} \mathrm{H}$ ratio to $\alpha_{\mathrm{IR}}$, indicating that there is no evolutionary sequence within our sample. Instead observed variations in the $\mathrm{CH}_{3} \mathrm{OH} / \mathrm{C}_{4} \mathrm{H}$ ratio in our sample (visible in Figure 6 as a large spread in $\mathrm{CH}_{3} \mathrm{OH}$ column densities for any specific $\mathrm{C}_{4} \mathrm{H}$ column density) seems to be the result of different initial ice compositions.

Figure 7 shows a positive correlation between the $\mathrm{C}_{4} \mathrm{H} / \mathrm{CH}_{3} \mathrm{OH}$ gas and the ice phase $\mathrm{CH}_{4} / \mathrm{CH}_{3} \mathrm{OH}$. The correlation is not statistically significant due to the many ice upper limits, however it does suggest a relationship between gas and ice abundances for these species. When $\mathrm{C}_{4} \mathrm{H}$ gas and $\mathrm{CH}_{4}$ ice, and $\mathrm{CH}_{3} \mathrm{OH}$ ice and gas are considered separately, there seems to be a stronger relationship between the gas and ice phase hydrocarbons (Figure 7 bottom) than between $\mathrm{CH}_{3} \mathrm{OH}$ gas and ice (Figure7 middle).

\section{DISCUSSION}

In this study, we observed 16 embedded protostars to determine the relationship between $\mathrm{CH}_{3} \mathrm{OH}$ and $\mathrm{C}_{4} \mathrm{H}$. We find that there is a positive correlation between the column density of $\mathrm{CH}_{3} \mathrm{OH}$ and $\mathrm{C}_{4} \mathrm{H}$. Several of the sources have COM detections in addition to $\mathrm{CH}_{3} \mathrm{OH}$ (Oberg et al. 2014). WCCC and COM chemistry are thus not mutually exclusive during the embedded stages of star formation. Rather, carbon chain and COM formation seem to follow one another up until the onset of an efficient hot corino chemistry. Once the hot corino has formed, $\mathrm{CH}_{3} \mathrm{OH}$ and $\mathrm{C}_{4} \mathrm{H}$ are no longer correlated (Maret et al. 2005).

Aikawa et al. (2012) modeled the chemistry from a prestellar core up to the formation of the protostellar envelope, and found that both carbon chains and COMs can be present in the colder, outer envelope. Aikawa et al. (2012) attribute the formation of the carbon chains to an increase in the $\mathrm{C}^{+}$abundance in conjunction with the $\mathrm{CH}_{4}$ ice sublimation. The presence of $\mathrm{CH}_{3} \mathrm{OH}$ in the envelope is due to non-thermal sublimation of $\mathrm{CH}_{3} \mathrm{OH}$ ice. Contrary to $\mathrm{C}_{4} \mathrm{H}, \mathrm{CH}_{3} \mathrm{OH}$ is also abundant toward the protostellar core due to thermal sublimation. The abundance of $\mathrm{CH}_{3} \mathrm{OH}$ in the protostellar core can be many orders of magnitude higher than the outer envelope emission, but its contribution to observed emission can be quite negligible if the volume of thermally sublimated $\mathrm{CH}_{3} \mathrm{OH}$ is small compared to the protostellar envelope volume. Should the emission from the thermally sublimated $\mathrm{CH}_{3} \mathrm{OH}$ dominate the $\mathrm{CH}_{3} \mathrm{OH}$ envelope emission, the source would be considered a hot 

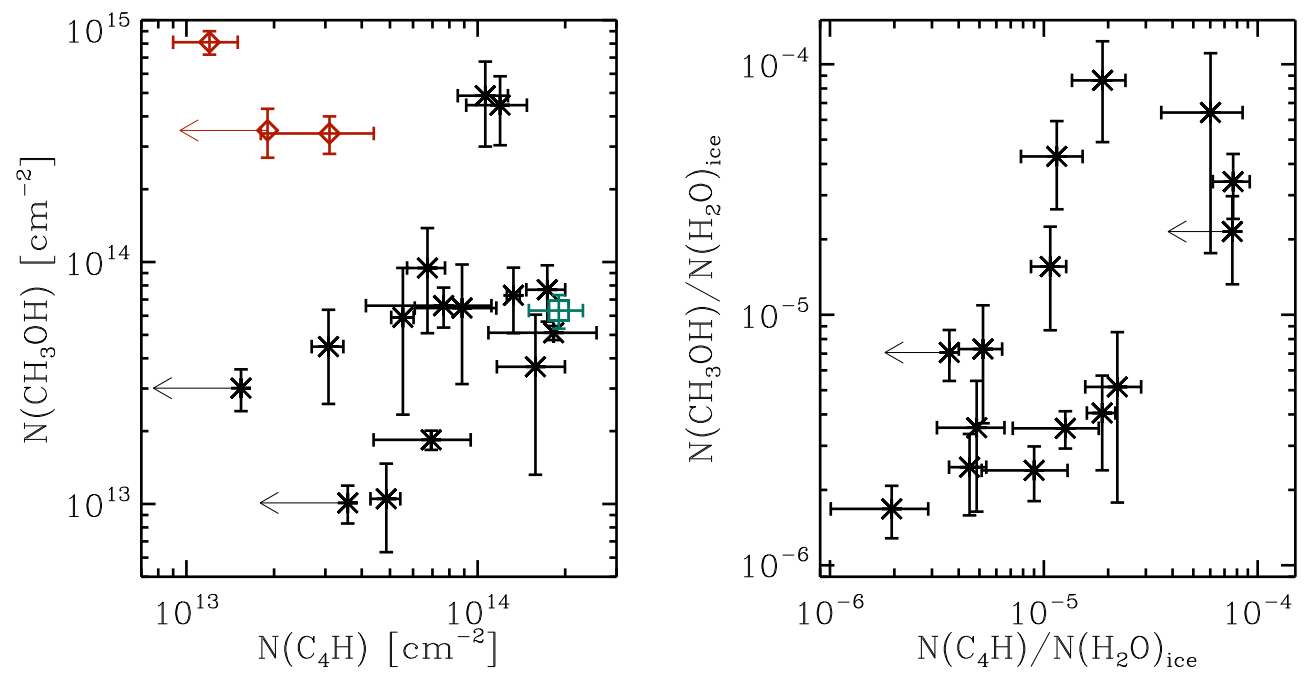

Figure 6. Correlation plots for the column density of $\mathrm{C}_{4} \mathrm{H}$ and $\mathrm{CH}_{3} \mathrm{OH}$ from our sample. The left plot displays the pure column density correlation where the black data points are our data, the teal square is L1527 (Sakai et al. [2008, 2009b), and the red diamonds are three hot corino sources (Maret et al. 2005; Sakai et al. 2009b). The right plot displays the $\mathrm{CH}_{3} \mathrm{OH}$ to $\mathrm{C}_{4} \mathrm{H}$ correlation following a normalization using the $\mathrm{H}_{2} \mathrm{O}$ ice column density.

corino.

Within our source sample, envelope ice abundances explain variations in the $\mathrm{C}_{4} \mathrm{H} / \mathrm{CH}_{3} \mathrm{OH}$ ratio. The $\mathrm{CH}_{3} \mathrm{OH}$ ice and gas columns (Figure 7 middle) are not clearly related, however. If all $\mathrm{CH}_{3} \mathrm{OH}$ in all sources originated from non-thermal desorption there should be a correlation between $\mathrm{CH}_{3} \mathrm{OH}$ gas and ice abundances. On the other hand if some $\mathrm{CH}_{3} \mathrm{OH}$ is due to thermal desorption, the source luminosity will influence the $\mathrm{CH}_{3} \mathrm{OH}$ emission, muddling the expected $\mathrm{CH}_{3} \mathrm{OH}$ gas and ice correlation. As we observe no correlation, it is likely that we are probing both thermal and non-thermal desorption processes.

The observed tentative $\mathrm{C}_{4} \mathrm{H}-\mathrm{CH}_{4}$,ice correlation indicates that the formation of $\mathrm{C}_{4} \mathrm{H}$ depends on the initial abundance of $\mathrm{CH}_{4}$ ice. The thermal sublimation of $\mathrm{CH}_{4}$ ice to promote WCCC was initially suggested by Sakai et al. (2008), and is consistent with current chemical models (Hassel et al. 2011; Aikawa et al. 2012). Not all $\mathrm{C}_{4} \mathrm{H}$ may originate from this process, however, as some may be inherited from the molecular cloud. An unusually high contribution of inherited $\mathrm{C}_{4} \mathrm{H}$, may explain the outlier with a high $\mathrm{C}_{4} \mathrm{H}$ gas column and low $\mathrm{CH}_{4}$ ice upper limit (IRAS 03271+3010) in Figure 7. A possible explanation for such a high contribution may be that this source is very young.

In addition to ice abundances, the chemistry of $\mathrm{C}_{4} \mathrm{H}$ and $\mathrm{CH}_{3} \mathrm{OH}$ may depend on environmental parameters, such as envelope mass and bolometric luminosity. A higher bolometric luminosity should result in a larger hot corino region. If this is the main regulator for the COMscarbon chain relationship, the $\mathrm{CH}_{3} \mathrm{OH} / \mathrm{C}_{4} \mathrm{H}$ ratio would increase toward more luminous protostars. We do not find any correlation between this ratio and source luminosity (Tables 1 and 4). It is more difficult to assess the importance of the initial envelope mass on the chemistry, since envelope mass evolves with time. A current low envelope mass measurement may signify either a low-mass protostar or an older more massive protostar. We do not find any correlation between the $\mathrm{CH}_{3} \mathrm{OH} / \mathrm{C}_{4} \mathrm{H}$ ratio and envelope mass alone (Tables 1 and 4). The small sample size prevented a more detailed analysis of the combined influence of mass and age on the chemistry. We also do not see any relationships between these environmental parameters and the absolute column densities of $\mathrm{C}_{4} \mathrm{H}$ and $\mathrm{CH}_{3} \mathrm{OH}$.

As reported in $\S 4$, we also find no correlation with $\alpha_{\mathrm{IR}}$, an age indicator, and the $\mathrm{CH}_{3} \mathrm{OH} / \mathrm{C}_{4} \mathrm{H}$ ratio. For young embedded protostars, the 'age' of the system does not seem to affect the relative importance of carbon chain and $\mathrm{COM}$ chemistry, i.e. $\mathrm{C}_{4} \mathrm{H}$ and $\mathrm{CH}_{3} \mathrm{OH}$ coexist in the envelope at this evolutionary stage. In Figure 6 , the "boomerang" shape may indicate an evolutionary trend when comparing these young objects with more evolved ones. This is consistent with models. As the protostar transitions into the hot corino stage, the temperature is high enough to both destroy carbon chains and thermally desorb $\mathrm{CH}_{3} \mathrm{OH}$, resulting in an anti-correlation in the observed $\mathrm{C}_{4} \mathrm{H}$ and $\mathrm{CH}_{3} \mathrm{OH}$ column densities Aikawa et al. 2012).

\section{CONCLUSIONS}

We surveyed 16 northern sources selected from the Spitzer $c 2 d$ ice sample using the IRAM $30 \mathrm{~m}$ and found the following:

1. $\mathrm{C}_{4} \mathrm{H}$ and $\mathrm{CH}_{3} \mathrm{OH}$ coexist and are correlated at the deeply embedded stage of low-mass protostellar evolution. This can be explained by lukewarm environments in the protostellar envelopes that promote WCCC alongside non-thermal desorption of $\mathrm{CH}_{3} \mathrm{OH}$.

2. The $\mathrm{CH}_{3} \mathrm{OH} / \mathrm{C}_{4} \mathrm{H}$ correlation does not extend into the hot corino phase. This is indicative of an evolutionary sequence where carbon chains and COMs coexist in lukewarm protostellar envelopes, but once a hot corino forms, COMs are enhanced and carbon chains are destroyed.

3. The ice and gas abundances are related in these sources based on a tentative correlation between $\mathrm{CH}_{3} \mathrm{OH} / \mathrm{C}_{4} \mathrm{H}$ gas ratio and $\mathrm{CH}_{3} \mathrm{OH} / \mathrm{CH}_{4}$ ice ratio. 

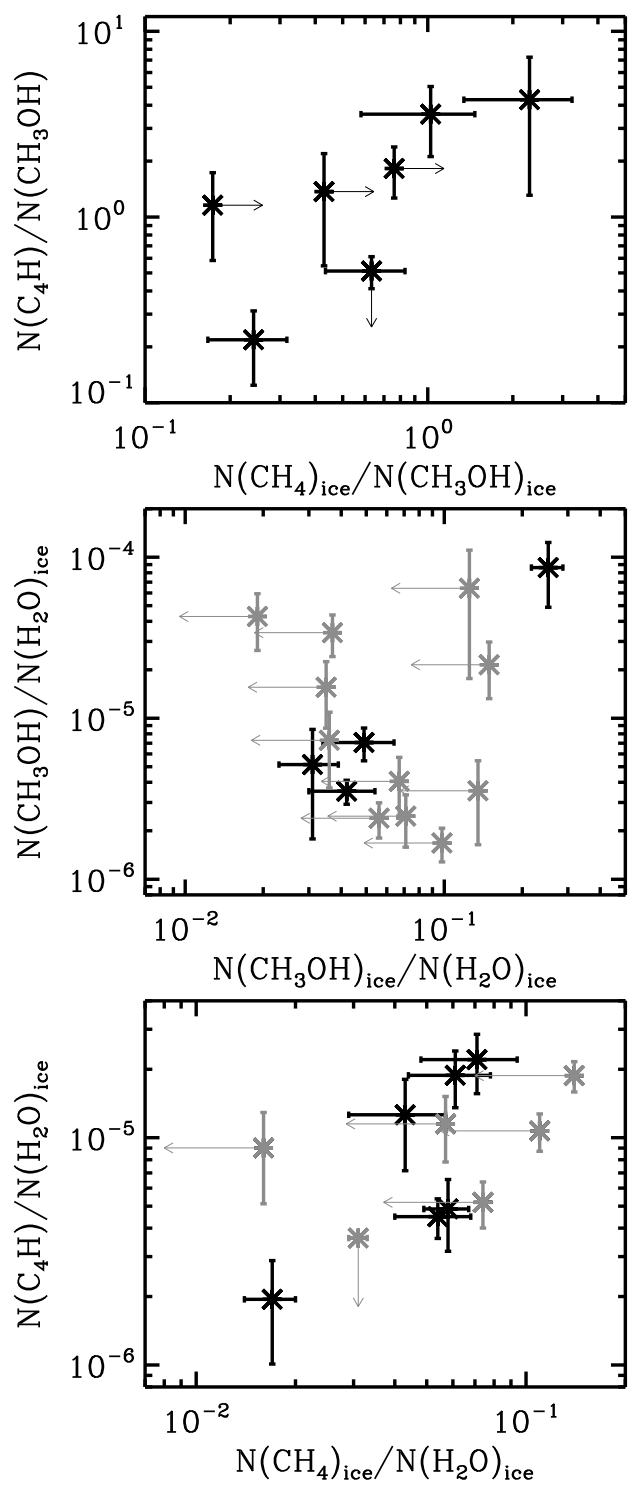

Figure 7. Ice and gas correlation plots from our sample. The top plot displays the $\mathrm{CH}_{4}$ to $\mathrm{CH}_{3} \mathrm{OH}$ ice ratio versus the $\mathrm{C}_{4} \mathrm{H}$ to $\mathrm{CH}_{3} \mathrm{OH}$ gas ratio. The middle plot displays the ice $\mathrm{CH}_{3} \mathrm{OH}$ versus the gas $\mathrm{CH}_{3} \mathrm{OH}$ columns, and the bottom plot displays the ice $\mathrm{CH}_{4}$ versus the gas $\mathrm{C}_{4} \mathrm{H}$ columns. Both of the bottom two plots are normalized to the $\mathrm{H}_{2} \mathrm{O}$ ice column density and the gray data points indicate the upper limits.

It is likely that the $\mathrm{C}_{4} \mathrm{H}$ gas and $\mathrm{CH}_{4}$ ice correlation is what is driving this relationship, suggesting that WCCC is intimately connected to the $\mathrm{CH}_{4}$ ice abundance.

This work has benefited from discussions with Ryan Loomis, Viviana Guzman, Edith Fayolle, and from the helpful comments of the anonymous referee. The study is based on observations with the IRAM 30m Telescope. IRAM is supported by INSU/CNRS (France), MPG (Germany) and IGN (Spain). KIÖ acknowledges funding from the Simons Collaboration on the Origins of Life Investigator award \#321183, the Alfred P. Sloan Foun- dation, and the David and Lucile Packard Foundation.

\section{REFERENCES}

Aikawa, Y., Wakelam, V., Hersant, F., Garrod, R. T., \& Herbst, E. 2012, ApJ, 760, 40

Andre, P., Ward-Thompson, D., \& Barsony, M. 2000, Protostars and Planets IV, 59

Arce, H. G., Santiago-García, J., Jørgensen, J. K., Tafalla, M., \& Bachiller, R. 2008, ApJL, 681, L21

Arce, H. G. \& Sargent, A. I. 2006, ApJ, 646, 1070

Bacmann, A., Taquet, V., Faure, A., Kahane, C., \& Ceccarelli, C. 2012, A\&A, 541, L12

Balucani, N., Ceccarelli, C., \& Taquet, V. 2015, MNRAS, 449, L16

Blake, G. A., Sutton, E. C., Masson, C. R., \& Phillips, T. G. 1987, ApJ, 315, 621

Boogert, A. C. A., Gerakines, P. A., \& Whittet, D. C. B. 2015, ARA\&A, 53, 541

Boogert, A. C. A., Pontoppidan, K. M., Knez, C., et al. 2008, ApJ, 678, 985

Bottinelli, S., Boogert, A. C. A., Bouwman, J., et al. 2010, ApJ, 718,1100

Brinch, C., Crapsi, A., Hogerheijde, M. R., \& Jørgensen, J. K. 2007, A\&A, 461, 1037

Broten, N. W., Oka, T., Avery, L. W., MacLeod, J. M., \& Kroto, H. W. 1978, ApJL, 223, L105

Carter, M., Lazareff, B., Maier, D., et al. 2012, A\&A, 538, A89

Cazaux, S., Tielens, A. G. G. M., Ceccarelli, C., et al. 2003, ApJL, 593, L51

Cernicharo, J., Marcelino, N., Roueff, E., et al. 2012, ApJL, 759, L43

Chen, H., Grenfell, T. G., Myers, P. C., \& Hughes, J. D. 1997, ApJ, 478, 295

Enoch, M. L., Evans, II, N. J., Sargent, A. I., \& Glenn, J. 2009, ApJ, 692, 973

Evans, II, N. J., Allen, L. E., Blake, G. A., et al. 2003, PASP, 115,965

Furlan, E., McClure, M., Calvet, N., et al. 2008, ApJS, 176, 184

Garrod, R. T. \& Herbst, E. 2006, A\&A, 457, 927

Garrod, R. T., Weaver, S. L. W., \& Herbst, E. 2008, ApJ, 682, 283

Goldsmith, P. F. \& Langer, W. D. 1999, ApJ, 517, 209

Hassel, G. E., Harada, N., \& Herbst, E. 2011, ApJ, 743, 182

Hatchell, J., Fuller, G. A., Richer, J. S., Harries, T. J., \& Ladd, E. F. 2007, A\&A, 468, 1009

Herbst, E. \& Leung, C. M. 1989, ApJS, 69, 271

Herbst, E. \& van Dishoeck, E. F. 2009, ARA\&A, 47, 427

Kroto, H. W., Kirby, C., Walton, D. R. M., et al. 1978, ApJL, 219, L133

Little, L. T., Riley, P. W., \& Matheson, D. N. 1977, MNRAS, $181,33 \mathrm{P}$

Maret, S., Ceccarelli, C., Tielens, A. G. G. M., et al. 2005, A\&A, 442,527

Öberg, K. I., Boogert, A. C. A., Pontoppidan, K. M., et al. 2008, ApJ, 678, 1032

Öberg, K. I., Bottinelli, S., Jørgensen, J. K., \& van Dishoeck, E. F. 2010, ApJ, 716, 825

Öberg, K. I., Bottinelli, S., \& van Dishoeck, E. F. 2009a, A\&A, 494, L13

Öberg, K. I., Garrod, R. T., van Dishoeck, E. F., \& Linnartz, H. 2009b, A\&A, 504, 891

Öberg, K. I., Lauck, T., \& Graninger, D. 2014, ApJ, 788, 68

Ohishi, M. \& Kaifu, N. 1998, Faraday Discussions, 109, 205

Pontoppidan, K. M., van Dishoeck, E. F., \& Dartois, E. 2004, A\&A, 426, 925

Sakai, N., Sakai, T., Hirota, T., Burton, M., \& Yamamoto, S. 2009a, ApJ, 697, 769

Sakai, N., Sakai, T., Hirota, T., \& Yamamoto, S. 2008, ApJ, 672, 371

Sakai, N., Sakai, T., Hirota, T., \& Yamamoto, S. 2009b, ApJ, 702,1025

Sakai, N. \& Yamamoto, S. 2013, Chemical Reviews, 113, 8981

Wilking, B. A., Bontemps, S., Schuler, R. E., Greene, T. P., \& André, P. 2001, ApJ, 551, 357 